\title{
Non-linearity variation of single junctions and its analysis using nano- prober within fine-grained ZnO-based multilayer varistors
}

\author{
Eiichi Koga, Device Solution Business Unit, Automotive \& Industrial Systems Company, Panasonic Co., Ltd., 1037-2, Kamiosatsu, Chitose City, Hokkaido \\ 066-8502, Japan \\ Yoshiko Higashi, Device Solution Business Unit, Automotive \& Industrial Systems Company, Panasonic Co., Ltd., 1006 Kadoma, Kadoma City, Osaka \\ 571-8506, Japan
}

Address all correspondence to Eiichi Koga at koga.eiichi@jp.panasonic.com

(Received 23 March 2016; accepted 28 June 2016)

\begin{abstract}
Direct electrical measurement of single grain boundaries is performed for ZnO-based multilayer ceramic varistors with fine grains of $2 \mu \mathrm{m}$, using a nano-prober. The effect of $\mathrm{ZnO}$ grains on non-linearity is shown to be significant. The microstructure is comprised at least two non-linear types as good- and bad-junctions. The numerical ratio of good to bad is estimated to be about one (non-linearity exponent $\left.\alpha_{\max } \geq 10\right)$ to two $(<10)$, which is independent of microstructural development as grain growth. The grain control of twinning as well as crystal orientation and donor concentration is considered effective in the further improvement.
\end{abstract}

\section{Introduction}

$\mathrm{ZnO}$-based ceramic varistors exhibit highly non-linear currentvoltage characteristics, and are utilized extensively as electrical circuit protectors against power and voltage surges ${ }^{[1]}$ Recently, to protect ICs in mobile and automotive devices from an electro static discharge (ESD) and a load dump surge (LSD), Bi- or Pr-doped ZnO-based multilayer ceramic varistors (MLCVs) are widely employed, due to their very good protection performance combined with small size. ${ }^{[2]}$ To assist high integration and high performance of the ICs, to further enhance protection performance as well as reliability is strongly required for MLCVs in future. ${ }^{[2-4]}$ Moreover, MLCVs using base metal electrodes have also been studied recent years to dramatically reduce the internal electrode cost in current MLCVs cofired with precious metals (e.g., Pd, Pt, and $\mathrm{Ag} / \mathrm{Pd}$ ). ${ }^{[5]}$ To use base metals as internal electrodes, they have to be sintered in a reducing atmosphere so as not to cause oxidation of them, showing non-linearity and the electrical stability. Non-linearity of varistors originates from a grain-boundary region between $\mathrm{ZnO}$ grains in the microstructure so that it is very sensitive to variations of the oxidation state and composition around grain boundaries by thermal treatment as sintering or electrical stress as voltage surges. ${ }^{[3,5]}$ Thus, knowledge of structural and electrical details of the grain boundary is intrinsically important in improving the performance of MLCVs. Conventionally, electrical barriers of the grain boundaries have been evaluated on the basis of Schottky barrier model such as $C-V$ analysis. ${ }^{[6]}$

A previous error in this article's header has been corrected.
However, because of the electrical properties as polycrystalline materials with many grain boundaries, individual grain boundaries within the microstructure could not be characterized from the measurement of varistors as a device. Meanwhile, the electrical property of single grain boundaries in varistors with very large grains of about $150 \mu \mathrm{m}$ is reported by M. Tao, which were produced by use of seed grain method. ${ }^{[7]}$ They have observed both good and bad barriers in non-linear $V-I$ curves in the microstructure. However, recent commercial MLCVs are composed of fine grains as about $2 \mu \mathrm{m}$ in thin varitor-layers of thickness below $20 \mu \mathrm{m}$, to bring low-voltage characteristics (e.g., 5.5-12 V). ${ }^{[2,3]}$ Therefore, to enhance ESD/LDS protection performance and reliability for future MLCVs, further research is desirable to understand the electrical characteristics of single grain boundaries in fine-grained $\mathrm{ZnO}$ varistors.

In the present study, direct measurement of the electrical property of single-grain boundaries is undertaken to resolve the issues for fine-grained MLCVs. In it, we describe the variation in electrical behavior of different grain-to-grain junctions on the local structure, using a nano-prober system for integrated semiconductor devices. Consequently, high-level uniformity of individual junctions by $\mathrm{ZnO}$ grain control as twinning, crystal orientation, and donor concentration, is shown to be intrinsically essential for improvement of performance of MLCVs.

\section{Experimental procedure}

All of the samples used in the present study were prepared by the solid-state reaction method. Our samples were produced from reagent grade powders of $\mathrm{ZnO}$ and additives. $\mathrm{ZnO}$-based varistors in this paper were produced using $\mathrm{ZnO}$ 

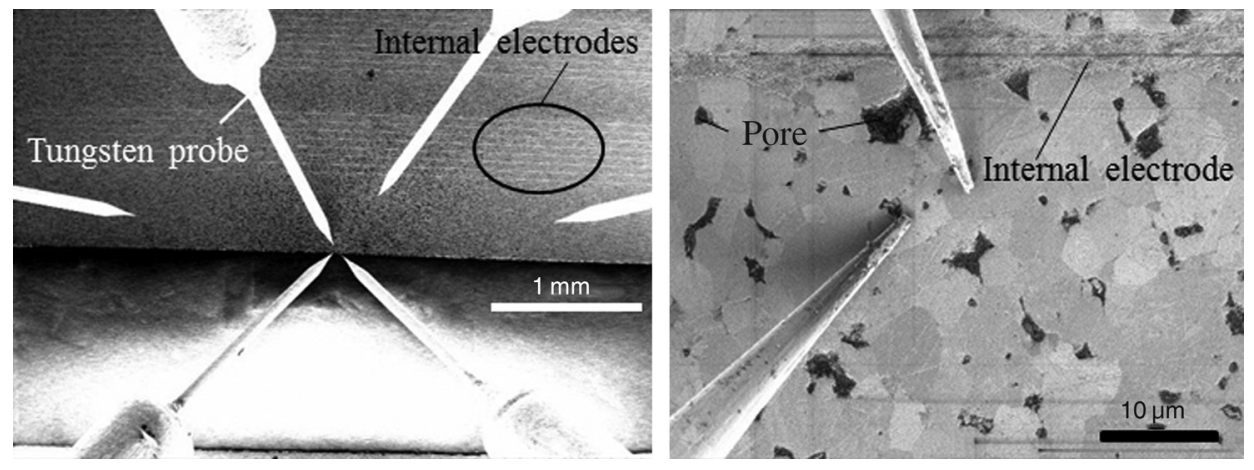

Figure 1. SEM images of direct measurement of a single junction between $\mathrm{ZnO}$ grains using a nano-prober system.

containing $\mathrm{Bi}_{2} \mathrm{O}_{3}, \mathrm{Sb}_{2} \mathrm{O}_{3}$, and small amounts of other elements, such as $\mathrm{Co}_{2} \mathrm{O}_{3}, \mathrm{MnO}_{2}, \mathrm{NiO}, \mathrm{Cr}_{2} \mathrm{O}_{3}, \mathrm{SiO}_{2}, \mathrm{Al}_{2} \mathrm{O}_{3}$, and $\mathrm{B}_{2} \mathrm{O}_{3}$. The composition is a typical commercial formulation for practical varistors. Fabricated MLCVs were produced using the conventional multilayer ceramic process. Details on the synthesis condition of MLCVs are given in our previous papers. ${ }^{[3]}$ The green chips were constructed into $8.5 \mathrm{~mm}$ in length, 7.5 $\mathrm{mm}$ in width, and $4.0 \mathrm{~mm}$ in height. They have a structure in which, 30 varistor-layers and Ag-internal electrodes are alternately stacked. The sintered thickness of varistor-layers is about $50 \mu \mathrm{m}$ between the internal electrodes. They were sintered at about $930{ }^{\circ} \mathrm{C}$ below the melting point of $\mathrm{Ag}\left(960^{\circ} \mathrm{C}\right)$ in air. The resulting sintered chips were then terminated with Ag-based electrodes at $800{ }^{\circ} \mathrm{C}$ in air, and we finally obtained MLCVs of a size of 5.5, 5.0, and $3.0 \mathrm{~mm}$ in length, width, and height. Their $V-I$ properties are evaluated using DC current from $1 \mu \mathrm{A}$ to $1 \mathrm{~mA}$, and voltages at the measured currents were defined as $V_{1 \mu \mathrm{A}}, V_{10 \mu \mathrm{A}}, V_{100 \mu \mathrm{A}}$, and $V_{1 \mathrm{~mA}}$, respectively. Here, $V_{1 \mathrm{~mA}}$ is usually termed varistor voltage, meaning breakdown voltage as a device. The non-linearity exponents $\alpha_{10 \mu \mathrm{A}}$ were determined from $V-I$ data, between current of $10 \mu \mathrm{A}$ and $1 \mathrm{~mA}$, according to the following equation: $\alpha_{10 \mu \mathrm{A}}=\left(\log I_{1 \mathrm{~mA}}-\log \right.$ $\left.I_{10 \mu \mathrm{A}}\right) /\left(\log V_{1 \mathrm{~mA}}-\log V_{10 \mu \mathrm{A}}\right)$. MLCVs studied have $V_{1 \mathrm{~mA}}=$ $27 \mathrm{~V}, \alpha_{10 \mu \mathrm{A}}=20$ and capacitance $=30 \mathrm{nF}$ at $1 \mathrm{kHz}$. Varistor voltage per a grain boundary $\left(V_{\mathrm{gb}}\right)$ in MLCVs was determined from $V_{1 \mathrm{~mA}}$ and an average grain size, which is measured by the intercept method from the microstructural observation [scanning electron microscope (SEM) images] of the cross-sectional surface in the same way as given in our previous paper. ${ }^{[5]}$ In addition, for electrically microstructural analysis, MLCVs were ground to approximately the mid plane with SiC papers and polished carefully with finer alumina powders. After polishing and drying, dry etching of $\mathrm{CaF}_{2}$ was performed for the polished surface of them. Under a SEM, one tungsten probe was directly contacted with each surface of two adjacent $\mathrm{ZnO}$ grains. It is confirmed experimentally that the contact between the $\mathrm{ZnO}$ grain and tungsten probe has no resistance $(\approx 0 \Omega)$, from the measurement using two probes in the surface of a $\mathrm{ZnO}$ grain. Electrical measurements of $V-I$ behavior were then conducted for single-grain junctions between two adjacent $\mathrm{ZnO}$ grains using a nano-prober system, as shown in Fig. 1. The $V$ - I measurement were performed at least two times to confirm superior data reproducibility. The dada were then analyzed in the present study.

\section{Results and discussion $V-I$ characteristics for single junctions at grain boundaries around a ZnO grain}

$V-I$ behavior for three adjacent single junctions were measured across grain boundaries around a $\mathrm{ZnO}$ grain within the MLCV shown in Fig. 2. The results implied that direct measurement using a nano-prober is very available for the microstructural analysis of electrical properties in fine-grained varistors. Shown in Fig. 3 is $V-I$ characteristics for the individual single junctions at each of three grain boundaries around a $\mathrm{ZnO}$ grain. Different behavior of $V-I$ curve are clearly found to be obtained by changing only one side of the $\mathrm{ZnO}$ grain. The results on adjacent $\mathrm{ZnO}$ grains reveal that the three grain to grain junctions within the local microstructure (i.e., around a $\mathrm{ZnO}$ grain) possess not same but different $V-I$ characteristics with the wide non-linearity variation of good to bad junctions. The junction area between grains not being known due to having a large variation of grain size in the microstructure, we are not able to similarly analyze them for an equal current density. Therefore, non-linearity of a single junction in the present study also was evaluated on the basis of the method by the former study. ${ }^{[7]}$ The maximum non-linear exponent $\left(\alpha_{\max }\right)$ is determined from $\alpha$-values $\left[\alpha=\left(\log I_{2}-\log I_{1}\right) /\left(\log V_{2}-\log V_{1}\right)\right]$ in $V-I$ property. A voltage corresponding to resulting $\alpha_{\max }$ is defined as $V_{\alpha \max }$, namely, a breakdown voltage at a grain boundary. $V_{\alpha \max }$ and $\alpha_{\max }$ as non-linear index were thus characterized for each single junction. It is quantitatively shown from the result that the three junctions between the adjacent grains have considerable variations in $V_{\alpha \max }$ of 2.1-2.7 and $\alpha_{\max }$ of 5.4-34.0 as summarized in Table I. The large variation of $V-I$ behavior for the three junctions is found in finegrained MLCVs as well as that of varistors consisting of large grains $(150 \mu \mathrm{m})$ in the previous report. ${ }^{[7]}$ It is possible to say that the effect of $\mathrm{ZnO}$ grains on $V-I$ property is very significant despite adjacent grains within narrow region. The electrical characteristics for a grain boundary has been analyzed for 


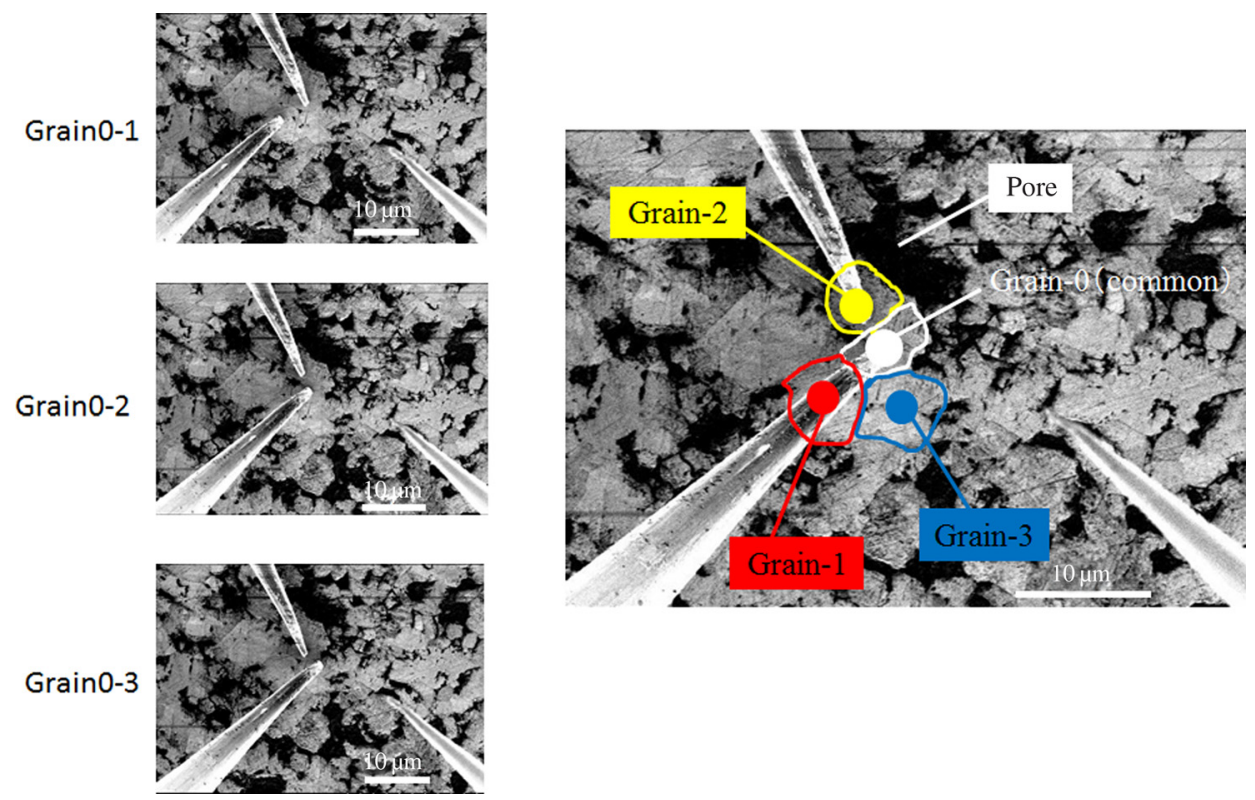

Figure 2. SEM images when measuring for three adjacent single junctions across grain boundaries around a ZnO grain within the MLCV.

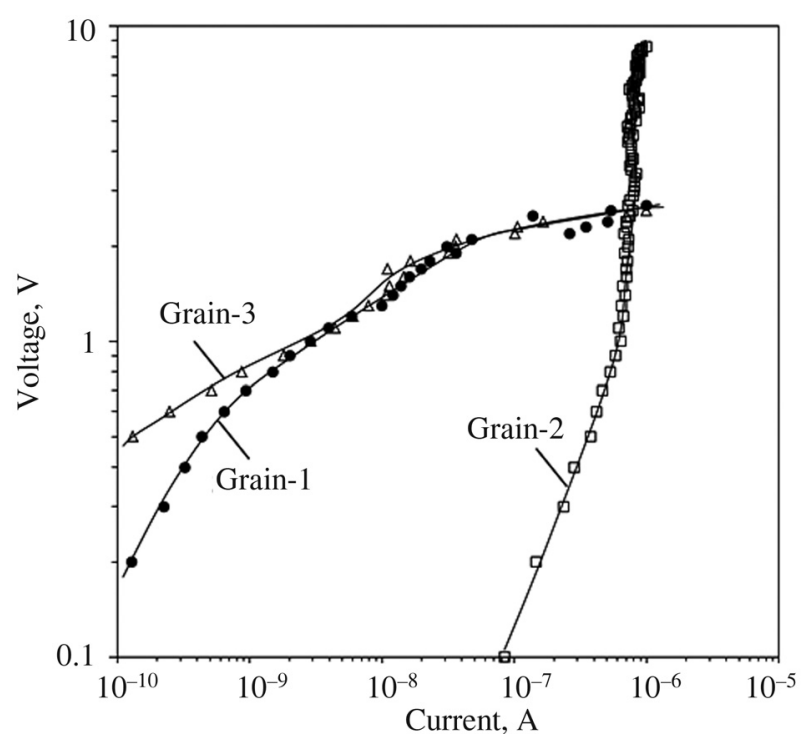

Figure 3. $V-/$ curves of three single junctions between the common grain-0 and around the grain $(-1,2$, and 3$)$.

Table I. $V_{\alpha \max }$ measured using nano-prober system and apparent $V_{\mathrm{gb}}$ determined by $V_{1 \mathrm{~mA}}$ and grain boundary numbers.

\begin{tabular}{lll}
\hline \hline$V_{\alpha \max }$ & $1.78 \mathrm{~V}$ & Nano-prober system \\
\hline$\alpha_{\max }$ & 8.71 & \\
\hline$V_{\mathrm{gb}}$ & $1.98 \mathrm{~V}$ & $V_{1 \mathrm{~mA}} /$ grain-boundary numbers \\
\hline
\end{tabular}

the simplified model of the boundary between bi-crystals joined by two $\mathrm{ZnO}$ single crystals. ${ }^{[8,9]}$ The results reported in previous studies have demonstrated that the property of a single junction is able to be significantly influenced by the atomic structural relationship between two grains and by the electrical states (i.e., donor density) of each grain. ${ }^{\left[{ }^{7-9}\right]}$ Thus, the different $V-I$ characteristics of the junctions around a $\mathrm{ZnO}$ grain probably is closely related to crystal orientation and/or donor concentrations of $\mathrm{ZnO}$ grains across a grain boundary. In addition, the grains in $\mathrm{Bi}$-doped $\mathrm{ZnO}$ varistors consist of not only single crystal, but also twining, containing a very small amount of Co and donor (i.e., $\left.\mathrm{Al}^{3+}\right) .{ }^{[10,11]}$ The presence of twined grains substructure has been reported to cause high non-linearity, because of other Schottky-type energy barriers comprising at twin grain boundaries. ${ }^{[12,13]}$ Hence, the non-uniform behavior of three individual grain boundaries as shown in Fig. 3 may be attributed as well to the microstructure combined with two grain types of single and twinning crystal. However, the origin of non-linearity variation in single junctions of adjacent grains has yet to be fully elucidated in the present study. This will need to be further investigated by means of precise crystal-structural and electrical analysis of the microstructure.

\section{Variation in non-linearity of single junctions in MLCVs}

$V-I$ curves of single junctions $(N=30)$ selected randomly in the microstructure were examined for the fine-grained MLCV. The arithmetic means of $V_{\alpha \max }$ and $\alpha_{\max }$ by the direct measurement are presented in Table I, with $V_{\mathrm{gb}}\left(=V_{1 \mathrm{~mA}} /\right.$ numbers of grain boundaries) given from $V_{1 \mathrm{~mA}}$ as a device and the numbers of microstructural grain boundaries. In some cases, current 

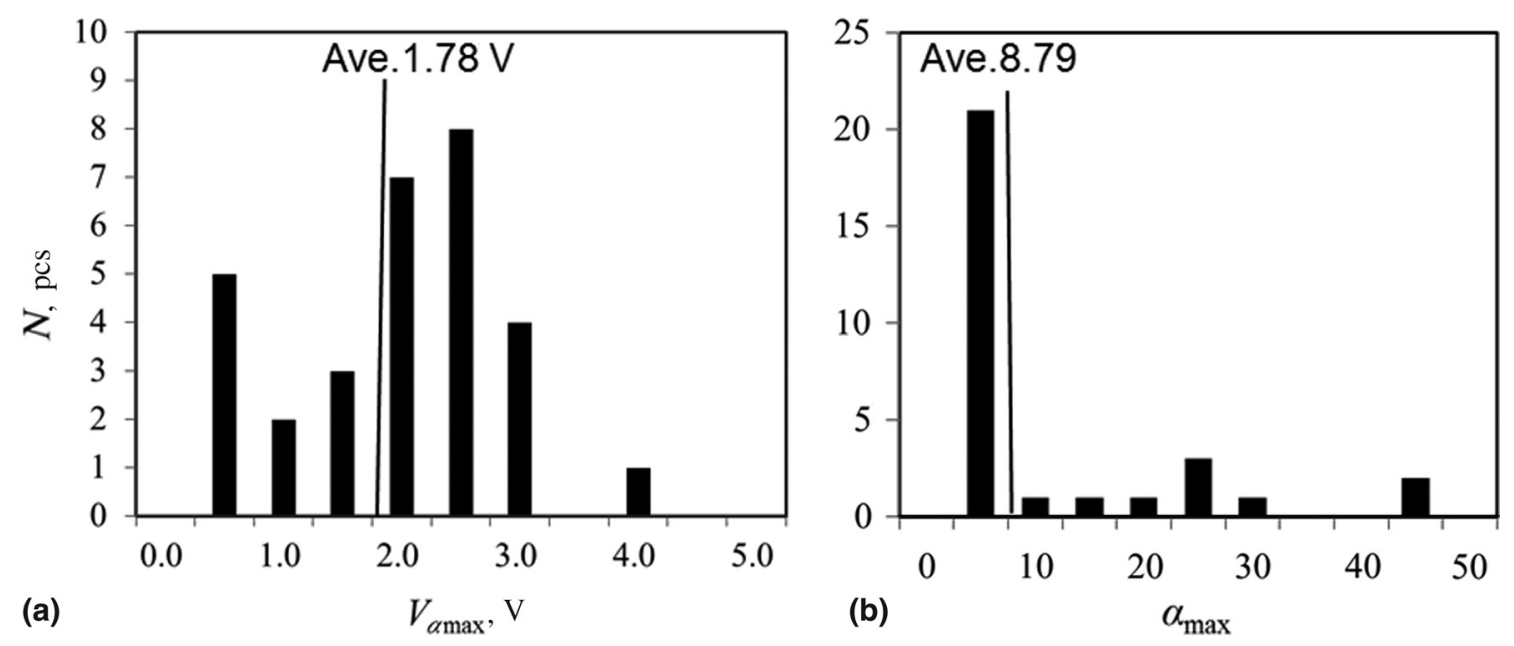

Figure 4. Frequency distributions of $V_{\alpha \max }$ and $\alpha_{\max }$ [(a) $V_{\alpha \max }$ and (b) $\alpha_{\max }$ ].

through a back side of the measured grain surface may be detected in the present evaluation, thus we have compared with breakdown voltages at a grain boundary given by the different measurement method. It is evident from Table I that $V_{\alpha \max }$ $(=1.78 \mathrm{~V})$ and $V_{\mathrm{gb}}(=1.98 \mathrm{~V})$ agree with each other because the difference between them is $<10 \%$. It has been confirmed from the result that they are in good agreement with each other regardless of the different measurement methods. Thus, we can say that direct measurement using a nano-prober system is a very useful means for electrical analysis of individual single junctions within fine-grained varistors. Further detailed examination of non-linear characteristics was then performed to characterize $V_{\alpha \max }$ and $\alpha_{\max }$ by the direct measurement. The frequency distributions of $V_{\alpha \max }$ and $\alpha_{\max }$ are presented in Fig. 4 [(A) $V_{\alpha \max }$ and (B) $\alpha_{\max }$ ], respectively. It should be noted that the results apparently demonstrate that both of the distributions are not only wide, but also asymmetrical in histograms. The wide asymmetry is suggested to result from the presence of several non-linear components in the microstructure. Similar distributions to $V_{\alpha \max }$ and $\alpha_{\max }$ in finegrained MLCVs are observed also in the results of $\mathrm{ZnO}$ varistors with large grains $(150 \mu \mathrm{m})$, which were provided by the different measurement means using wire contacts from the present. The previous report has indicated that $\mathrm{ZnO}$ varistors with large grains have the wide non-linearity variation of good to bad junctions in the polycrystalline. Similarly, non-linear property of fine-grained MLCVs was found comprised their junctions with large variations in $V_{\alpha \max }(=0.3-2.8 \mathrm{~V})$ and $\alpha_{\max }$ $(=1.1-44.0)$ as illustrated in histograms. Totaling of $V-I$ characteristics for individual junctions given by a nano-prober system should be able to present the non-linear characteristics of MLCVs as a device. Hence, it is likely that a mean value of $V_{\alpha \max }(=1.78 \mathrm{~V})$ by the direct measurement would be good agreement with $V_{\mathrm{gb}}(=1.98 \mathrm{~V})$, which was estimated from the property of MLCVs and microstructural observation.

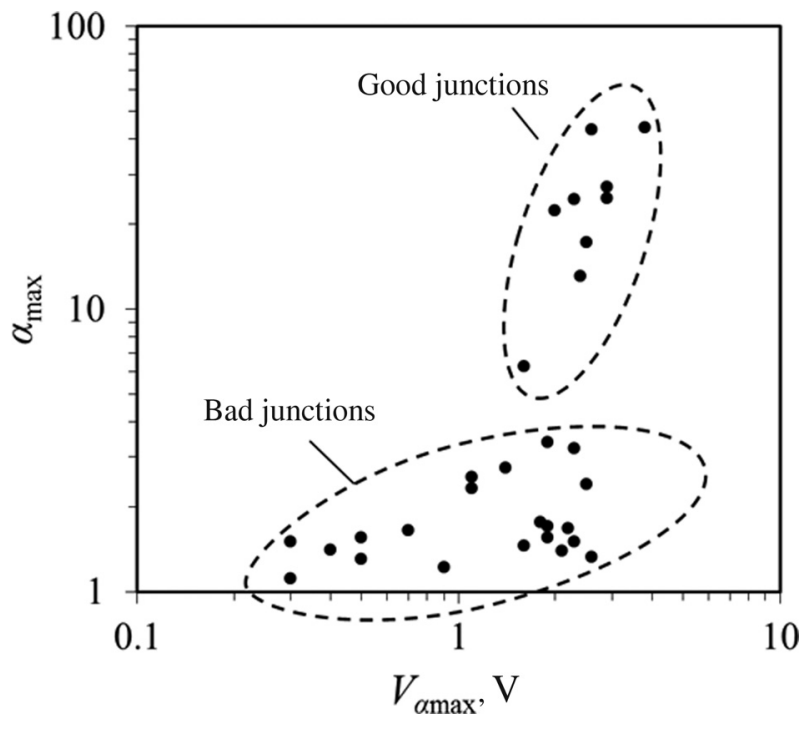

Figure 5. Scatter diagram for $V_{\alpha \max }$ versus $\alpha_{\max }$ of single junctions.

To further examine their specific property in frequency distributions, a scatter diagram was plotted for $V_{\alpha \max }$ versus $\alpha_{\max }$. The result is shown in Fig. 5. It appears that the resulting relationship is able to be distinguished between at least two groups as good and bad junctions. Good junctions with high nonlinearity of $\alpha_{\max }$ (e.g., $\geq 10$ ) are found to have relatively high $V_{\alpha \max }$ levels of about 1.8-2.8 V. On the other hand, bad junctions showing low $\alpha_{\max }$ (e.g., $<10$ ) seem to be composed of a lower $V_{\alpha \max }$ range of 0.3-2.5 V. From their results, it is apparent that individual junctions in MLCVs are not uniform characteristics, irrespective of whether the microstructure of varistors is fine grained with an average size of $2 \mu \mathrm{m}$ or not. The microstructure of MLCVs studied seems to have at least two types of good and bad junctions as mentioned above, and we are able to 
quote about 2.0 and $1.4 \mathrm{~V}$ as their mean values in the distributions, respectively. The difference of $V_{\alpha \max }$ between them is clearly significant. From the numbers of two types, the numerical ratio of good to bad junctions is estimated to be about one to two within the present MLCVs, which is seen to be in rough agreement with that of the result shown in the previous study on varistors with large grains. ${ }^{[7]}$ The results imply that the ratio of good to bad junctions probably is independent of microstructural development as grain growth. The two types of junction characteristics are believed to be due to different barrier levels comprising the presence of two grain types (single and twinning) rather than effects of crystal orientation and donor concentrations of $\mathrm{ZnO}$ grains, as described above. However, the effect of grain structures on non-linearity of single junctions is not yet fully clarified; detailed investigation is further required.

In general, both non-linearity and stability against ESD are degraded with decreasing varistor voltage by reducing the number of grain boundaries. ${ }^{[2,3]}$ The characteristic degradations with decreasing grain-boundary density should occur due to forming a low resistance path for electrical current to flow between internal electrodes. The similar path, which is continuously connected by bad junctions with large leakage current, would be able to affect not only non-linearity, but also the stability of MLCVs. Needless to say, the presence of bud junctions in the polycrystalline provides the decrement of nonlinearity as MLCVs. Moreover, microstructural current densities should undoubtedly become extremely high concentration in the path region (i.e., bad junctions), thereby causing degradation of stability.

From results obtained, we thus believe that in electrical a sense, high-level uniformity of single junctions is intrinsically essential for improvement of protection performance and reliability rather than the fine-grained microstructure. Accordingly, decreasing non-linearity variation as bad junctions should play the crucial role in further characteristic improvement of MLCVs. The grain control of twinning as well as crystal orientation and donor concentration is considered effective in enhancing the non-linearity uniformity. There would be probability of further influence on the electrically microstructural control, by texture engineering using templated grain growth or magnetic field, sintering process such as millimeter heating, and synthesis from mechanically treated nano-composite particles. ${ }^{[14-17]}$

\section{Conclusions}

In the present work, direct measurement of the electrical property of individual grain boundaries was performed for finegrained MLCVs using a nano-prober system. Our findings from the experimental analysis are listed below. (i) Direct measurement using a nano-prober system is very available for the microstructural analysis of the electrical property in fine-gained varistors. (ii) The effect of $\mathrm{ZnO}$ grains on $V-I$ property is very significant. The non-linearity variation probably is independent of microstructural development as grain growth. (iii) The microstructure of fine-grained MLCVs was found to be comprised of at least two non-linear types of good and bad junctions. The two junction characteristics are believed to be due to different barrier levels comprising the presence of two grain types (single and twinning) rather than effects of crystal orientation and donor concentrations of $\mathrm{ZnO}$ grains. Good junctions with high non-linearity of $\alpha_{\max }$ (e.g., $\geq 10$ ) possess relatively high $V_{\alpha \max }$ of 1.8-2.8 V. On the other hand, bad junctions showing low $\alpha_{\max }$ (e.g., <10) seem to be composed of a lower $V_{\alpha \max }$ range of $0.3-2.5 \mathrm{~V}$. The numerical ratio of good to bad junctions was estimated to be about one to two within the MLCVs studied.

In conclusion, it was found that individual single junctions in MLCVs are not uniform characteristics, regardless of fine grained, owing to non-linearity variation at grain boundaries in the polycrystalline. The experimental analysis implies that decreasing bad junctions of small non-linear coefficient is essential to enhance performance of MLCVs. The grain control of twinning as well as crystal orientation and donor concentration probably is effective in significantly improving performance of MLCVs and the electrical stability against ESD/LDS. However, until now, no work on varistors would have focused on the grain control for enhancement of the practical performance as a protection device. We therefore believe that to study the grain control technology within varistors should be an important theme for further improvement of MLCVs in the future.

\section{References}

1. M. Matsuoka: Nonohmic properties of zinc oxide ceramics. Jpn. J. Appl. Phys. 10, 736 (1971).

2. E. Koga, N. Sawada, M. Amisawa, S. Minami, and T. Okimoto: Multilayer ceramic chip varistors with low varistor voltage for ESD-protection. Panasonic Tech. J. 58, 53-58 (2012).

3. E. Koga and N. Sawada: Electrical degradation caused by electro-static discharge pulse in ZnO-based multilayer varistor. Key Eng. Mater. 388, 15 (2009).

4. E. Koga and N. Sawada: Multilayer varistor with low-voltage characteristics from $\mathrm{ZnO}+\mathrm{ACOO}_{3}$ ceramics $(\mathrm{A}=\mathrm{Ca}$, Sr and Ba). Key Eng. Mater. 485, 249-252 (2011).

5. Y. Higashi, M. Hogiri, and E. Koga: Nonlinear property of $\mathrm{SrCoO}_{3}$-doped $\mathrm{ZnO}$ ceramics sintered in a reducing atmosphere and multilayer ceramic varistors. J. Mater. Res. 30, 2300-2309 (2015).

6. K. Mukae, K. Tsuda, and I. Nagasawa: Capacitance-vs-voltage characteristics of Zn0 varistors. J. Appl. Phys. 50, 4475-4476 (1979).

7. M. Tao, B. Ai, O. Dorlanne, and A. Loubiere: Different "single grain junctions" within a ZnO varistor. J. Appl. Phys. 61, 1562 (1987).

8. Y. Nakamura, T. Harada, H. Kuribara, A. Kishimoto, N. Motohira, and $H$. Yanagida: Nonlinear current-voltage characteristics with negative resistance observed at $\mathrm{ZnO}-\mathrm{ZnO}$ single-contacts. J. Am. Ceram. Soc. 82 , 3069-3074 (1999).

9. Y. Sato, T. Yamamoto, and Y. Ikuhara: Atomic structures and electrical properties of ZnO grain boundaries. J. Am. Ceram. Soc. 90, 337-357 (2007)

10. Y.-M. Chiang and W.D. Kingery: Compositional changes adjacent to grain boundaries during electrical degradation of a $\mathrm{ZnO}$ varistor. J. Appl. Phys. 53, 1765-1768 (1982).

11.M. Inada: Microstructure of nonohmic zinc oxide ceramics. Japan. J. Appl. Phys. 17, 673-677 (1978).

12. B.A. Haskell, S.J. Souri, and M.A. Helfand: Varistor behavior at twin boundaries in ZnO. J. Am. Ceram. Soc. 82, 2106-2110 (1999). 
13. P. Duran, J. Tartaj, and C. Moure: Fully dense, fine-grained, doped zinc oxide varistors with improved nonlinear properties by thermal processing optimization. J. Am. Ceram. Soc. 86, 1326-1329 (2003).

14. K. Wada, K. Kakimoto, and H. Ohsato: Control of temperature coefficient of resonant frequency in $\mathrm{Ba}_{4} \mathrm{Sm}_{9.33} \mathrm{Ti}_{18} \mathrm{O}_{54}$ ceramics by templated grain growth. Sci. Technol. Adv. Mater. 6, 54-60 (2005).

15. S. Tanaka, A. Makiya, Z. Kato, and N. Uchida: Fabrication of C-axis oriented polycrystalline $\mathrm{ZnO}$ by using a rotating magnetic field and following sintering. J. Mater. Res. 21, 703-707 (2006).

16. W.-C. Lee, K.-S. Liu, and I.-N. Lin: Nonlinear electrical properties of ZnO varistors fast-fired by using millimeter-wave sintering process. J. Mater. Sci. 35, 4841-4847 (2000).

17. S. Tasaki, J. Tatami, H. Nakano, T. Wakihara, K. Komeya, and T. Meguro: Fabrication of $\mathrm{ZnO}$ ceramics using $\mathrm{ZnO} / \mathrm{Al}_{2} \mathrm{O}_{3}$ nanocomposite particles prepared by mechanical treatment. J. Ceram. Soc. Jpn. 118, 118-121 (2010). 